\title{
A NOTE CONCERNING THE DISTANCES OF UNIFORMLY DISTRIBUTED POINTS FROM THE CENTRE OF A RECTANGLE
}

\author{
ROBERT STEWART ${ }^{\bowtie}$ and HONG ZHANG
}

(Received 11 February 2012; accepted 29 April 2012)

\begin{abstract}
Given a rectangle containing uniformly distributed random points, how far are the points from the rectangle's centre? In this paper we provide closed-form expressions for the cumulative distribution function and probability density function that characterise the distance. An expression for the average distance to the centre of the rectangle is also provided.
\end{abstract}

2010 Mathematics subject classification: primary 60D05; secondary $68 \mathrm{~T} 40$.

Keywords and phrases: rectangle, distribution of distances, probability density function, cumulative distribution function, mean distance, rectangle picking, robotic computer vision.

\section{Introduction}

In this paper we provide closed-form solutions for the cumulative distribution function (CDF), probability density function (PDF) and expected value for the distance to the centre of a rectangle of uniformly distributed random points (within the rectangle).

The motivation for the work presented here stems originally from our research in appearance-based simultaneous localisation and mapping (SLAM) in the field of mobile robotics, where we have been developing methods for assessing image similarity that enable a robot, equipped with a vision sensor (camera), to determine if it has arrived at a new location or returned to a previously visited location. As part of our research, we were investigating a scenario where uniformly distributed random points in a camera image move towards the centre of the image (as the camera recedes from a scene) [19] and became interested in the possibility of mathematically characterising the distance these points moved. It was this scenario that provided the impetus for the work presented in this current paper.

In the literature a formula for the average distance to the centre of a square has been reported (see, for example, [8]) and is related to the problem of 'square

The authors would like to gratefully acknowledge partial funding of this work by NSERC. Robert Stewart would additionally like to acknowledge the support of a Postdoctoral Fellowship from the University of Alberta while he was on leave without pay from an Australian research organisation.

(C) 2012 Australian Mathematical Publishing Association Inc. 0004-9727/2012 \$16.00 


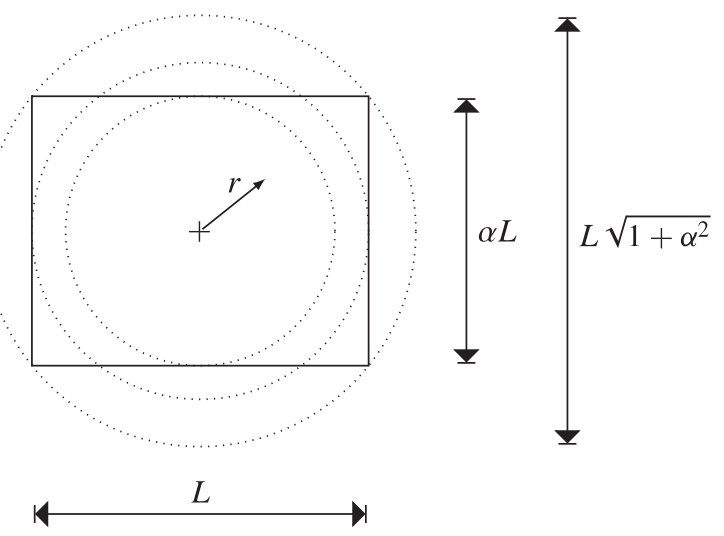

FIGURE 1. Relevant dimensions for a rectangle and for circles that define the limits on intervals of interest.

point picking' [21]. A number of works concerning the distance between two random points within and between rectangles $[5,9-13,16]$ and/or other geometric figures [1, 2, 4, 6, 7, 17] have also been published, with recent interest in similar and related topics being application-driven (for example, [3, 12-15, 18, 22]). However, surprisingly, there exist no closed-form expressions for the CDF, PDF and expected value for the distance of uniformly distributed random points to the centre of a rectangle, to the best of our knowledge. This paper aims to address this absence through the provision of such expressions. The results are of a fundamental nature so should have application beyond our initial field of inquiry.

\section{Derivation of the cumulative distribution and probability density functions}

In this section, an expression for the cumulative distribution function for the distances of uniformly distributed points from the centre of a rectangle is derived, and this leads to an expression for the probability density function.

Consider a rectangle with side lengths $L$ and $\alpha L$ as depicted in Figure 1, where $0<\alpha \leq 1$. By quantifying the overlap between the rectangle and a circle of radius $r$ positioned at the rectangle's centre, $(L / 2, \alpha L / 2)$, the area of the rectangle can be expressed as a function of $r$ :

$$
A(r)= \begin{cases}0, & r \leq 0, \\ A_{1}(r), & 0<r \leq \frac{\alpha L}{2}, \\ A_{2}(r), & \frac{\alpha L}{2}<r \leq \frac{L}{2}, \\ A_{3}(r), & \frac{L}{2}<r \leq \frac{L \sqrt{1+\alpha^{2}}}{2}, \\ \alpha L^{2}, & r>\frac{L \sqrt{1+\alpha^{2}}}{2},\end{cases}
$$


where

$$
\begin{gathered}
A_{1}(r)=\pi r^{2}, \\
A_{2}(r)=A_{1}(r)-2 r^{2} \cos ^{-1}\left(\frac{\alpha L}{2 r}\right)+\alpha L \sqrt{r^{2}-\left(\frac{\alpha L}{2}\right)^{2}}, \\
A_{3}(r)=A_{2}(r)-2 r^{2} \cos ^{-1}\left(\frac{L}{2 r}\right)+L \sqrt{r^{2}-\left(\frac{L}{2}\right)^{2}} .
\end{gathered}
$$

The cumulative distribution function, $F(r)$, or the probability that a uniformly distributed point is within a distance $r$ from the centre of the rectangle, is proportional to the area. Normalising (2.1), the CDF is therefore

$$
F(r)=\frac{A(r)}{\alpha L^{2}} .
$$

From (2.2), the probability density function can be found by taking the derivative of the CDF [20]. Using standard differentiation techniques, the CDF evaluates to:

$$
\begin{aligned}
f(r) & =\frac{d F(r)}{d r} \\
& = \begin{cases}0, & r \leq 0, \\
\frac{2 \pi r}{\alpha L^{2}}, & 0<r \leq \frac{\alpha L}{2}, \\
\frac{1}{\alpha L^{2}}\left(2 \pi r-4 r \cos ^{-1}\left(\frac{\alpha L}{2 r}\right)\right), & \frac{\alpha L}{2}<r \leq \frac{L}{2}, \\
\frac{1}{\alpha L^{2}}\left(2 \pi r-4 r \cos ^{-1}\left(\frac{\alpha L}{2 r}\right)-4 r \cos ^{-1}\left(\frac{L}{2 r}\right)\right), & \frac{L}{2}<r \leq \frac{L \sqrt{1+\alpha^{2}}}{2} \\
0, & r>\frac{L \sqrt{1+\alpha^{2}}}{2} .\end{cases}
\end{aligned}
$$

which can more concisely be written as

$$
f(r)=\frac{1}{\alpha L^{2}}\left(2 \pi r-4 r \operatorname{Re}\left(\cos ^{-1}\left(\frac{\alpha L}{2 r}\right)+\cos ^{-1}\left(\frac{L}{2 r}\right)\right)\right),
$$

such that the arguments to the inverse cosine terms may give nonreal (complex) results.

To graphically illustrate the probability density function, an example plot is shown in Figure 2 for parameter values of $L=1$ and $\alpha=0.8$.

\section{Expected value for the probability density function}

An expected value, $E[R]$, can be calculated from the probability density function using [20]:

$$
E[R]=\int_{-\infty}^{\infty} r f(r) d r
$$




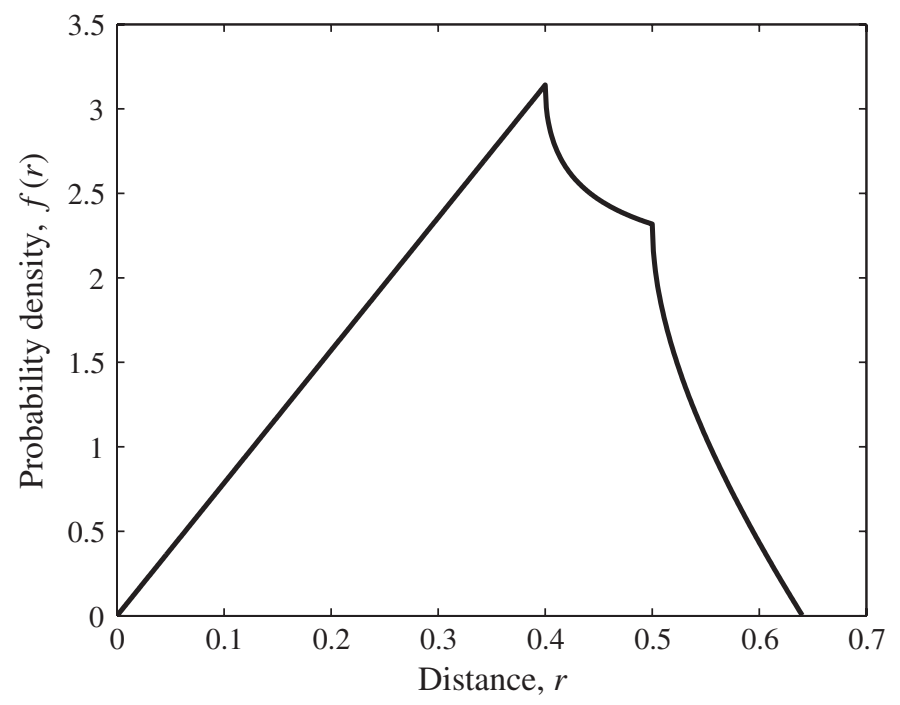

FIGURE 2. Example plot of the PDF for a rectangle with side lengths $L=1$ and $\alpha L=0.8$.

By substituting (2.3) into (3.1), the expected distance of a uniformly distributed random point to the centre of a rectangle can be found through standard integration techniques. The derivations are somewhat lengthy, so we report only the final result below:

$$
E[R]=\frac{L}{6}\left(\sqrt{1+\alpha^{2}}+\log \left(\left(\frac{\sqrt{1+\alpha^{2}}+1}{\sqrt{1+\alpha^{2}}-1}\right)^{\alpha^{2} / 4} \cdot\left(\frac{\sqrt{1+\alpha^{2}}+\alpha}{\sqrt{1+\alpha^{2}}-\alpha}\right)^{1 / 4 \alpha}\right)\right)
$$

Setting $L=1$ and $\alpha=1$ in (3.2), we obtain the commonly known result for a unit square [8]:

$$
E[R]=\frac{1}{6}(\sqrt{2}+\log (1+\sqrt{2})) \approx 0.3826 .
$$

It is worth noting that expression (3.2) for the expected value can be obtained (without need for the PDF) by solving the following double integral with an approach based on that used in [12] (and utilising lemmas therein):

$$
E[R]=\frac{1}{\alpha L^{2}} \int_{0}^{\alpha L} \int_{0}^{L} \sqrt{\left(x-\frac{L}{2}\right)^{2}+\left(y-\frac{\alpha L}{2}\right)^{2}} d x d y .
$$

\section{Acknowledgements}

The authors would like to thank family and colleagues for useful discussions regarding the work. Particular thanks to Christopher Stewart for providing some useful references and feedback on various aspects of the work. The authors would also like to thank an anonymous referee for providing a helpful reference and for making suggestions for improving the paper. 


\section{References}

[1] V. S. Alagar, 'The distribution of the distance between random points', J. Appl. Probab. 13 (1976), 558-566.

[2] B. Burgstaller and F. Pillichshammer, 'The average distance between two points', Bull. Aust. Math. Soc. 80 (2009), 353-359.

[3] D. P. Chu and A. R. Fotouhi, 'Distance between bivariate beta random points in two rectangular cities', Commun. Stat. Simul. Comput. 38 (2009), 257-268.

[4] M. F. Dacey, 'Some properties of order distance for random point distributions', Geogr. Ann.: Series B, Human Geography 49 (1967), 25-32.

[5] D. J. Daley, 'Solution to problem 75-12. An average distance', SIAM Rev. 18 (1976), 498-499.

[6] S. R. Dunbar, 'The average distance between points in geometric figures', College Math. J. 28 (1997), 187-197.

[7] P. F. Ehlers and E. G. Enns, 'Random secants of a convex body generated by surface randomness', J. Appl. Probab. 18 (1981), 157-166.

[8] S. R. Finch, Mathematical Constants (Cambridge University Press, Cambridge, 2003).

[9] B. Ghosh, 'On random distances between two rectangles', Sci. Cult. 8 (1943), 464.

[10] B. Ghosh, 'On the distribution of random distances in a rectangle', Sci. Cult. 8 (1943), 388.

[11] B. Ghosh, 'Random distances within a rectangle and between two rectangles', Bull. Calcutta Math. Soc. $\mathbf{4 3}$ (1951), 17-24.

[12] D. M. Lazoff and A. T. Sherman, 'An exact formula for the expected wire length between two randomly chosen terminals', Technical Report, University of Maryland Baltimore County, 1994.

[13] D. M. Lazoff and A. T. Sherman, 'Problem 95-6. Expected wire length between two randomly chosen terminals', SIAM Rev. 38 (1995), 321-324.

[14] L. E. Miller, 'Distribution of link distances in a wireless network', J. Res. Natl. Inst. Stand. Technol. 106 (2001), 401-412.

[15] J. P. Mullen, 'Robust approximations to the distribution of link distances in a wireless network occupying a rectangular region', Mobile Comput. Commun. Rev. 7 (2003), 80-91.

[16] J. D. Murchland, 'Comment to problem 75-12. An average distance', SIAM Rev. 18 (1976), 498.

[17] T. K. Sheng, 'The distance between two random points in plane regions', Adv. Appl. Probab. 17 (1985), 748-773.

[18] S. Srinivasa and M. Haenggi, 'Distance distributions in finite uniformly random networks: theory and applications', IEEE Trans. Veh. Technol. 59 (2010), 940-949.

[19] R. L. Stewart and H. Zhang, 'Image similarity from feature-flow for keyframe detection in appearance-based SLAM', Proc. 2011 IEEE Int. Conf. on Robotics and Biomimetics (IEEEROBIO 2011), Phuket, Thailand, 7-11 December 2011, pp. 305-312.

[20] R. E. Walpole, R. H. Myers, S. L. Myers and K. Ye, Probability and Statistics for Engineers and Scientists, 8th edn (Pearson Prentice Hall, Upper Saddle River, NJ, 2007).

[21] E. W. Weisstein, 'Square point picking', MathWorld-A Wolfram Web Resource, http://mathworld.wolfram.com/SquarePointPicking.html.

[22] N. Wicker, 'A note on ball segment picking related to clustering', Pattern Recogn. Lett. 32 (2011), $651-655$.

ROBERT STEWART, Department of Computing Science, University of Alberta, Edmonton, Alberta, Canada T6G 2E8

e-mail: RLStewart@ieee.org

HONG ZHANG, Department of Computing Science, University of Alberta, Edmonton, Alberta, Canada T6G 2E8

e-mail: hzhang@ualberta.ca 